\title{
Sinonasal Ameloblastoma
}

\author{
Benjamin G. Barrena ${ }^{1} \cdot$ Billy J. Phillips ${ }^{1} \cdot$ Kenneth J. Moran ${ }^{2} \cdot$ Sasha J. Betz ${ }^{1}$
}

Received: 9 May 2018 / Accepted: 12 May 2018 / Published online: 30 May 2018

(c) This is a U.S. government work and its text is not subject to copyright protection in the United States; however, its text may be subject to foreign copyright protection 2018

\begin{abstract}
Ameloblastomas are benign but aggressive odontogenic tumors that most commonly affect the posterior mandible. Approximately $15 \%$ occur in the maxilla, with a subset thought to originate from the epithelial lining of the sinonasal cavities. Histologically, sinonasal ameloblastomas are identical to those of the oral cavity, with classical features of palisaded columnar basilar cells surrounding a central proliferation that resembles the stellate reticulum of a developing tooth. Unlike the gnathic variant, sinonasal ameloblastomas tend to affect males more than females, and the incidence of diagnosis peaks at a later age, approximately 60 years old. The overall prognosis is favorable, with local recurrence being the most common long-term sequalae.
\end{abstract}

Keywords Sinonasal ameloblastoma $\cdot$ Gnathic $\cdot$ Odontogenic neoplasm $\cdot$ Maxilla

\section{Case Description}

A 34 year-old male presented to a general dental clinic complaining of pain and swelling in his left upper jaw for a reported duration of 2-3 weeks. His medical history was non-contributory. The physical examination revealed cellulitis of the left upper face and a firm mass that crossed the midline intraorally. Additionally, the patient had poor oral hygiene and severe periodontal disease. A panoramic radiograph revealed a large radiolucent lesion in the left maxilla as well as multiple hopeless teeth. The patient was clinically diagnosed with an abscess and suspected concurrent neoplasm. Teeth 12, 13, and 15 were extracted under

Sasha J. Betz

sasha.j.betz.mil@mail.mil

Benjamin G. Barrena

benjamin.g.barrena.mil@mail.mil

Billy J. Phillips

billy.j.phillips8.mil@mail.mil

Kenneth J. Moran

kenneth.moran@tchealth.org

1 Naval Medical Center San Diego, 34800 Bob Wilson Drive, San Diego, CA 92134, USA

2 Indian Health Service Hospital, PO Box 600, Tuba City, AZ 86045, USA local anesthesia and the patient was placed on amoxicillin/ clavulanate acid. A CT scan was ordered.

\section{Radiographic Features}

The CT scan revealed a large, well-demarcated, heterogeneous soft tissue mass in the left maxillofacial region (Fig. 1). The epicenter of the lesion was in the left maxillary sinus, with anteromedial and superior extension to involve the left frontal sinus, ethmoidal air cells, and nasal cavity. It measured approximately $9.1 \mathrm{~cm}$ in anterior-posterior dimension, $9.7 \mathrm{~cm}$ superior to inferior, and $5.6 \mathrm{~cm}$ in width.

The lesion filled the entire left maxillary sinus, nasal cavity, and ethmoid sinus. It extended into the malar process, body of the zygoma, and floor of the orbit. Posterior involvement of the left body of the sphenoid, greater wing, pterygoid process, and medial and lateral pterygoid plates was noted. Mass effect and encroachment of the left pterygopalatine fossa and medial and lateral pterygoid muscles was associated with the sphenoidal involvement of the lesion. The tumor protruded through the left skull base along the sphenoid sinus. 
Fig. 1 3D volume rendering (upper left), axial (upper right), coronal (lower left), and sagittal (lower right) CT images without contrast. Red outline indicates the peripheral borders of the lesion. Red arrows indicate involvement of the left orbit and left cranial base

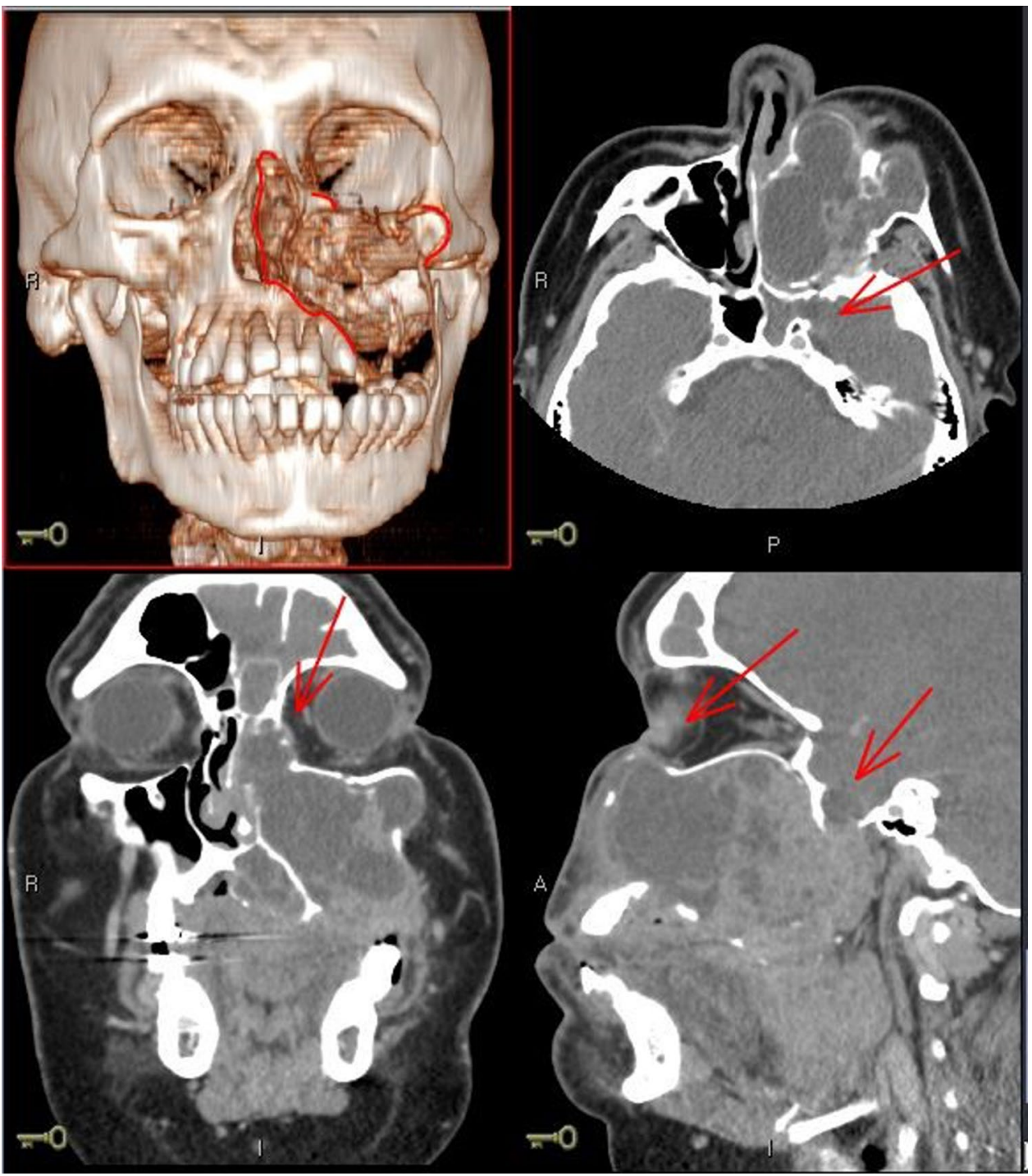

\section{Diagnosis and Treatment}

Following the CT scan, two biopsies of the mass were obtained under local anesthesia. The first biopsy consisted of a single portion of pink-red, rubbery tissue. The second biopsy was red-brown, irregular and unoriented.

Histologic examination revealed ameloblastic islands with columnar cells palisading around the periphery (Figs. 2, $3,4,5)$. The columnar cells demonstrated hyperchromatic nuclei and reverse polarity with subnuclear vacuoles. Within the center of these islands were loosely arranged angulated cells, resembling stellate reticulum. Numerous ameloblastic islands showed central squamous metaplasia. The patient was diagnosed with sinonasal ameloblastoma and referred for definitive treatment.

The patient underwent surgery which included left total ethmoidectomy, left frontal sinusotomy, left sphenoid

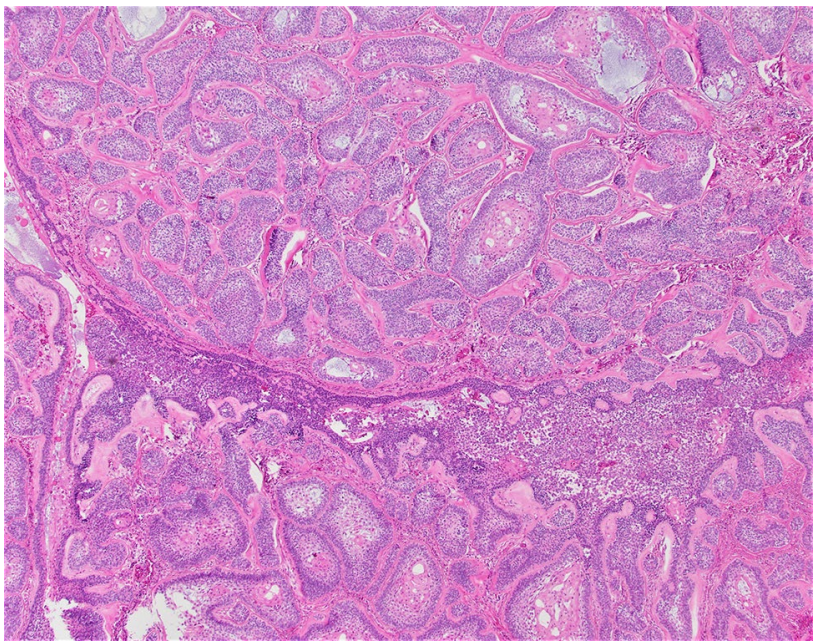

Fig. 2 Varying histologic patterns of ameloblastoma are appreciated at low power 


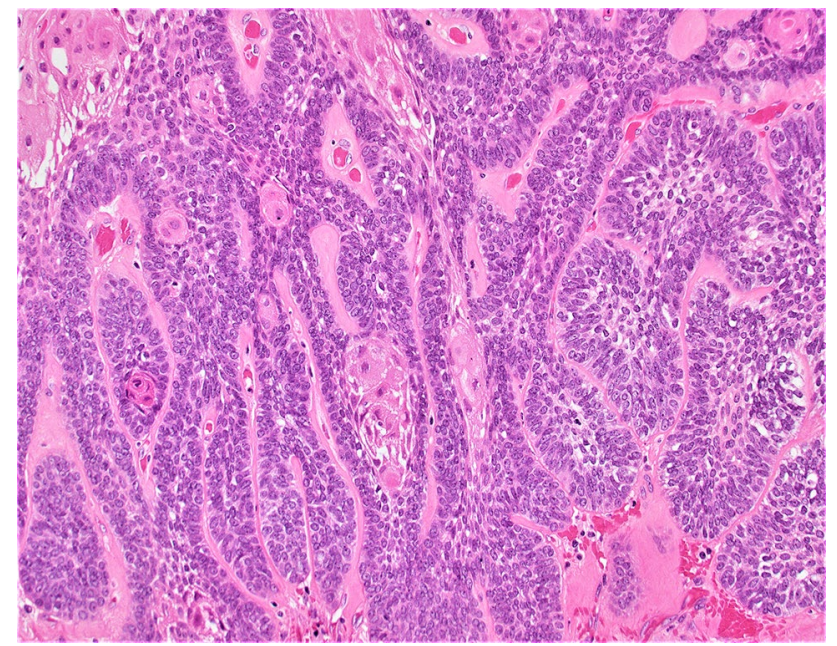

Fig. 3 An area of plexiform pattern demonstrating anastomosing cords of ameloblastic epithelium (high power)

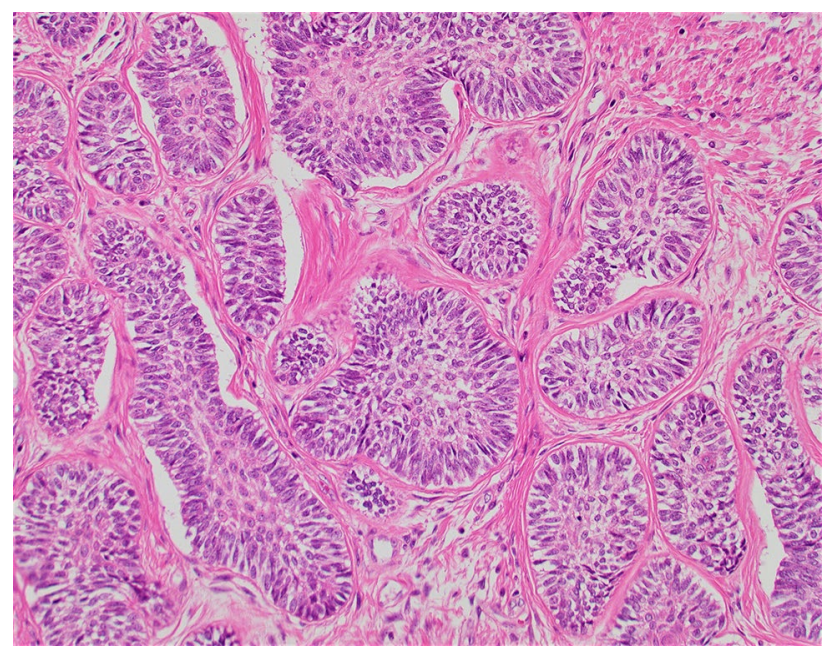

Fig. 4 An area demonstrating the follicular pattern. Reverse polarization and subnuclear vacuolization of the basal layer are appreciated (high power)

sinusotomy with tumor removal, left infratemporal fossa dissection for removal of tumor, free flap reconstruction, and left orbital reconstruction. The surgery was completed without complication and the patient was discharged on post-operative day 12 . The final pathology report confirmed the diagnosis of ameloblastoma with predominant plexiform, follicular, and acanthomatous patterns (Figs. 2, 3, 4, 5).

\section{Discussion}

Sinonasal ameloblastomas are rare tumors of the sinonasal tract that arise from sinonasal epithelium. A large review found ameloblastomas to comprise approximately $0.11 \%$

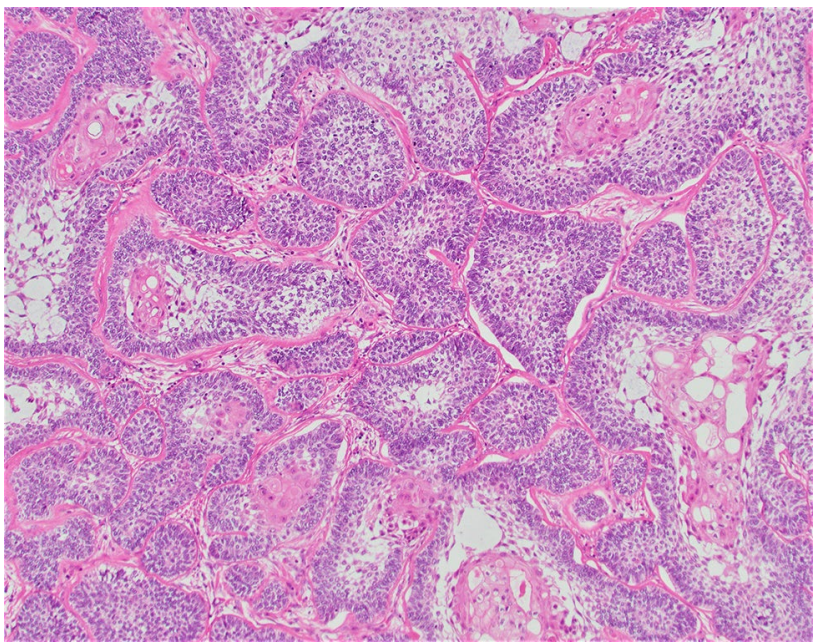

Fig. 5 Follicular and acanthomatous patterns dispersed within the same neoplasm (medium power)

$(n=19,658)$ of all sinonasal tract tumors [4]. In contrast to its gnathic counterpart, sinonasal ameloblastomas show a predilection for the male gender of 3.8:1 [4]. Additionally, these neoplasms present approximately $15-25$ years later than those occurring in the jaw, at an overall mean age of 59.7 versus $35-45$ years of age respectively $[1,4]$. Clinical presentation commonly includes nasal obstruction, sinusitis, and/or epistaxis [1-4]. Less common presenting signs and symptoms include facial swelling, headaches, and tearing [2-4].

Sinonasal ameloblastomas also differ from their counterparts in the jaw radiographically. Sinonasal lesions are most frequently described as radiopaque, solid lesions that fill the nasal cavity or sinus [4]. In contrast, ameloblastomas within gnathic areas commonly appear as radiolucent "honeycomb" or "bubble-like" lesions [1, 5]. Grossly, these primary sinonasal lesions are predominantly solid, grey-white, and rubbery to granular [4].

Histologically, sinonasal ameloblastoma resemble those lesions arising within the bones of jaw. The plexiform pattern is the most common pattern in sinonasal ameloblastomas, but the follicular pattern may also predominant [4]. The plexiform pattern of ameloblastoma is characterized by back-to-back anastomosing cords of epithelium, with peripheral columnar cells that demonstrate reverse polarity of their nuclei. Additionally, hyperchromasia of the nuclei and basal cytoplasmic vacuolization are readily identifiable features. Between the epithelial cords there are loosely arranged, angulated cells that comprise the stellate reticulum-like component of the tumor. Of note however, the stellate reticulum-like component of the tumor is less conspicuous in the plexiform pattern compared to the other subtypes of ameloblastoma [4]. Finally, transition from normal sinonasal tract epithelium to ameloblastomatous proliferation may be 
demonstrated microscopically and helps confirm the neoplasm is in fact primary to the sinonasal tract.

Surgical resection of primary sinonasal ameloblastomas is the treatment of choice [3,4]. Originally, radical surgery was performed on most patients given that treatment success heavily correlates with complete surgical removal of the lesion $[1,2]$. Conservative surgery or functional endoscopic sinus surgery (FESS) may be attempted in proper clinical setting, however complete removal of the tumor is the most important factor in determining the appropriate procedure $[3,4]$. The overall prognosis of sinonasal ameloblastomas is quite good with local recurrence being the most common long-term sequalae. Recurrence tends to occur within 1-2 years following surgery, however cases have been reported with recurrence occurring many years following surgery [1]. Of note, there has been no documented malignant transformation, metastasis or deaths attributed to sinonasal ameloblastomas $[2,4]$.

\section{References}

1. El-Naggar AK, Chan JKC, Grandis JR, Takata T, Slootweg PJ. WHO classification of head and neck tumours. 4th ed. Lyon: IARC; 2017.

2. Ereño C, Etxegarai L, Corral M, Basurko JM, Bilbao FJ, López JI. Primary sinonasal ameloblastoma. APMIS. 2005;113:148-50.

3. Kumar A, Sarswat S, Sharma S. Ameloblastoma presenting as a sinonasal tumor: rare entity. Int $\mathbf{J}$ Adv Health Sci. 2014;1(2):28-31.

4. Schafer D, Thompson L, Smith B, Wenig B. Primary ameloblastoma of the sinonasal tract: a clinicalpathologic study of 24 cases. Cancer. 1998;82(4):667-74.

5. Weissman JL, Snyderman CH, Yousem SA, Curtin HD. Ameloblastoma of the Maxilla: CT and MR appearance. ANJR. 1993;14:223-6. 